\title{
Fraturas de fêmur em idosos nas diferentes regiões do Brasil de 2015 a 2020: análise dos custos, tempo de internação e total de óbitos
}

\author{
Femur fractures in elderly in brazil from \\ 2015 to 2020: analysis of costs, time of \\ hospitalization and total deaths
}

\author{
Jefferson Carlos Araujo Silva ${ }^{1}$ (1) \\ Mara Dayanne Alves Ribeiro 2 (1) \\ Luan Nascimento da Silva ${ }^{3}$ (1)
}

\section{Hudson Azevedo Pinheiro 4 Lidia Mara Aguiar Bezerra 5 Sabrynna Brito Oliveira ${ }^{6}$ (1)}

\begin{abstract}
1'Autor para correspondência. Universidade de Brasília (Brasília). Distrito Federal, Brasil. jeffcasilva@gmail.com ${ }^{2}$ Hospital Regional do Norte (Sobral). Ceará, Brasil. mara_dayanne2@hotmail.com ${ }^{3}$ Hospital Escola da Universidade Federal de Pelotas (Pelotas). Rio Grande do Sul, Brasil. luan.nascimento2222@gmail.com ${ }^{4}$ Secretaria de Estado de Saúde do Distrito Federal (Brasília). Distrito Federal, Brasil. hudsonap@gmail.com 5Universidade de Brasília (Brasília). Distrito Federal, Brasil. lidia.bezerra@gmail.com ${ }^{6}$ Centro Universitário Metodista Izabela Hendrix (Belo Horizonte). Minas Gerais, Brasil. sabrynnabrito@gmail.com
\end{abstract}

RESUMO | INTRODUÇÃO: As fraturas de fêmur em idosos geram aumento da demanda funcional nesses indivíduos e são responsáveis por um alto número de internações hospitalares. OBJETIVO: Analisar retrospectivamente o total de casos, os custos, o tempo de internação e o total de óbitos por fratura de fêmur em idosos nas diferentes regiões do Brasil de 2015 a 2020. MÉTODOS: Estudo ecológico de série temporal e retrospectivo que avaliou os dados de fratura de fêmur em idosos, a partir de dados disponíveis nos sites de Ministério da Saúde. Foram avaliadas as seguintes variáveis: total de casos, Tempo Médio de Permanência Hospitalar (TMPH), número de óbitos, taxa de mortalidade, gastos com internações, total e por dia de internação hospitalar, Gasto Médio por Fratura (GMF) e o Tempo de Internação por Fratura (TIF) em cada região. RESULTADOS: Um total de 328.008 idosos sofreram fratura de fêmur no período estudado. A região Sudeste lidera em número de casos, óbitos e TIF; o segundo lugar alterna entre as regiões Sul (2015, 2016 e 2020) e Nordeste (2017 e 2019). Em relação ao TMPH, as regiões Norte e Nordeste aparecem em primeiro e segundo lugar, respectivamente, em todos os anos, ficando acima da média nacional em todos os anos estudados. CONCLUSÕES: Há uma discrepância para os casos de fratura de fêmur em idosos entre as regiões brasileiras, seja no número de casos, nos custos ou no total de óbitos. A maior densidade populacional em regiões como Sudeste e Sul, contribuiu para as maiores taxas de fraturas de fêmur em idosos, haja vista que estes também são maioria nessas regiões em comparação as demais.

PALAVRAS-CHAVE: Idoso. Fraturas do fêmur. Hospitalização. Custos de Cuidados de Saúde.

\begin{abstract}
INTRODUCTION: Femur fractures in the elderly generate increased functional demand for those who have and are responsible for a high number of hospital admissions. OBJECTIVE: Retrospectively analyze the total number of cases, costs, length of hospital stay, and total deaths from femoral fractures in the elderly in different regions of Brazil from 2015 to 2020. METHODS: Ecological study of temporal and retrospective series that evaluated fracture data of femur fracture in the elderly, based on data available on the websites of the Ministry of Health. The following variables were evaluated: total cases, Average Hospital Stay (AHS), number of deaths, mortality rate, hospitalization costs, total and per day of hospitalization, Average Fracture Cost (AFC), and Length of Hospitalization for Fracture (LHF) in each region. RESULTS: A total of 328,008 elderly people suffered a femur fracture during the study period. The Southeast region leads in cases, deaths, and LHF; the second-place alternates between the South $(2015,2016$, and 2020) and Northeast (2017 and 2019) regions. Concerning the AHS, the North and Northeast regions appear in first and second place, respectively, in all years, being above the national average in all years studied. CONCLUSIONS: There is a discrepancy for cases of femoral fractures in the elderly among Brazilian regions, whether in the number of cases, costs, or total deaths. The higher population density in regions such as the Southeast and the South contributes to the higher rates of femur fractures in the elderly, given that they are also the majority in these regions compared to the others.
\end{abstract}

KEYWORDS: Aged. Femoral Fractures. Hospitalization. Health Care Costs. 


\section{Introdução}

O envelhecimento populacional é uma realidade em países desenvolvidos e em desenvolvimento. No Brasil, o número total de idosos em 2020 era de 29,2 milhões, representando $14 \%$ do total da população, esse número deve chegar a 72,4 milhões no ano de 2100. ${ }^{1}$ Importante ressaltar que, por ser um país de dimensões continentais, o Brasil apresenta distribuição da população, estratégias e orçamento para prevenção de agravos e formas de atendimento da população de idosos muito variável entre as regiões brasileiras.?

O aumento exponencial da população idosa e a distribuição irregular pelo território nacional traz à tona questões relacionadas à esta parcela da população, o que nos faz refletir sobre medidas de prevenção e de assistência voltadas aos principais agravos que acometem essa população. Nos idosos, as Doenças Crônico Não Transmissíveis (DCNT) e fraturas ósseas estão entre os principais motivos de diminuição da qualidade de vida e mortes evitáveis. ํ․ $^{2}$

A população idosa é mais propensa a sofrer fraturas ósseas devido à perda de massa óssea e muscular decorrente do processo natural de envelhecimento. Além disso, os déficits de equilíbrio que tornam os idosos suscetíveis ao evento queda podem contribuir para uma incidência elevada de fraturas nesses sujeitos. ${ }^{4}$ As fraturas de fêmur em idosos são especialmente consideradas um grave problema de saúde pública em razão da alta letalidade e dos custos com seu tratamento. ${ }^{5}$

As fraturas de fêmur em idosos, habitualmente, decorrem de um trauma de baixa energia e possui íntima relação com a desnutrição, redução da mobilidade funcional, diminuição da acuidade visual, entre outros. ${ }^{6}$ As fraturas de fêmur na região proximal são mais comuns, incluindo as do colo do fêmur, transtrocantéricas e subtrocantéricas, na maioria dos casos há indicação de correção cirúrgica, porém, em casos específicos, como, fraturas incompletas, sem desvio ou diante da não condição clínica do paciente em ser submetido à cirurgia, o tratamento conservador pode ser indicado. ${ }^{\beth}$ As fraturas de fêmur são responsáveis pelo aumento da demanda funcional de idosos, além de sobrecarregar os familiares, que, na maioria das vezes, tornam-se cuidadores. - .

Inúmeras são as estratégias que visam prevenir as fraturas de fêmur em idosos, sejam medidas diretas ou indiretas, tais como estimular a prática regular de atividade física e a integração em grupos de convivência. Todas essas medidas objetivam a manutenção ou ganho de massa muscular, melhora do equilíbrio e da independência funcional, condições que indiretamente previnem a ocorrência de fraturas de fêmur. 5,9 No entanto, parece que essas medidas ainda não foram capazes de reduzir o número de idosos que sofrem fraturas de fêmur no Brasil.?

Tendo em vista as diferenças regionais observadas no Brasil, a hipótese do nosso estudo é de que há diferença nos custos, tempo de internação e total de óbitos por fratura de fêmur em idosos entre as regiões brasileiras. Diante disto, o objetivo do presente artigo foi analisar retrospectivamente o total de casos, os custos, o tempo de internação e o total de óbitos por fratura de fêmur em idosos de acordo com as regiões no Brasil, no período de 2015 a 2020.

\section{Métodos}

Foi realizado um estudo ecológico ${ }^{10}$ de série temporal e retrospectivo com dados dos casos de fratura de fêmur ocorridos em idosos no Brasil, no período de 2015 a 2020, a coleta de dados ocorreu entre fevereiro e março de 2021. A população do estudo foi constituída por todos os casos de fratura de fêmur ocorridos em idosos residentes no Brasil entre 2015 a 2020 e registrados no DATASUS, para obtenção dos dados seguiu-se os passos: DATASUS > ACESSO A INFORMAÇÃO > TABNET > SEÇÃO: EPIDEMIOLÓGICAS E DE MORBIDADE > Morbidade Hospitalar do SUS (SIH/SUS) > Geral, por local de internação - de 1984 a 2007 > Brasil por região e unidade da federação (http://tabnet.datasus.gov.br/cgi/ deftohtm.exe?sih/cnv/miuf.def) ${ }^{11}$ e utilizando os filtros: linha: região, conteúdo: internações ou óbitos, coluna: inativa, períodos disponíveis: 2010 a 2020. Lista Morb. CID-10: Fratura do fêmur. Foram utilizados os dados secundários referentes ao código S72 da Classificação Internacional de Doenças (CID-10). 
Foram avaliadas as seguintes variáveis: total de casos, Tempo Médio de Permanência Hospitalar (TMPH), número de óbitos, taxa de mortalidade, gastos com internações, total e por dia de internação hospitalar, Gasto Médio por Fratura (GMF) e o Tempo de Internação por Fratura (TIF) em cada região. A busca central se refere à fratura de fêmur e a comparação entre as regiões brasileiras.

O TMPH para fratura de fêmur em idosos foi calculado dividindo o total de dias de permanência no hospital pelo total de paciente internados, através da seguinte fórmula:

$\mathrm{TMPH}=($ Total de dias de permanência hospitalar por fratura de fêmur em idosos) / (Total de pacientes internados)

Os gastos do Sistema Único de Saúde (SUS) com as internações hospitalares foram analisados a partir do custo total, custo por dia de internação (CD) e gasto médio por fratura (GMF), este calculados por meio da fórmula a seguir:

GMF = (Tempo médio de permanência hospitalar por fratura de fêmur em idosos) / (Gasto total com fratura de fêmur em idosos )

$C D=($ Valor das despesas com internações por fratura de fêmur em idosos) / (Número total de dias de permanência por fratura de fêmur )

Foram feitos cálculos de frequência simples absoluta e relativa das variáveis do estudo. Por ser um estudo que incluiu dados de domínio público, não foi necessária a aprovação do Comitê de Ética em Pesquisa com seres humanos.

\section{Resultados}

Entre os anos 2015 e 2020, 328.008 idosos sofreram fratura de fêmur e foram atendidos pelo SUS no Brasil.
O número foi crescente de 2015 a 2019 (47.138, 52.130, $55.034,58.298,63.102$ de casos, respectivamente), tendo uma queda em 2020 (52.306 casos). Durante esse período foi observado que o TMPH dos idosos acometidos por fratura de fêmur e, consequentemente, os óbitos associados, variaram. De forma decrescente, os pacientes ficaram menos dias internados nos hospitais, sendo o TMPH de 9,1 dias nos anos de 2015 e 2016, 8,8 dias nos anos de 2017 e 2018, 8,5 dias em 2019 e 7,1 dias em 2020. Já o número de óbitos e taxa de mortalidade permaneceram crescentes nos três primeiros anos, caindo nos anos de 2019 e 2020 (Figura 1.A).

Os custos dessas fraturas ao SUS foi inversamente proporcional ao $\mathrm{TMPH}$, aumentando com o decorrer dos anos. A figura 1.B mostra o Gasto Total (GT) e o $C D$ das fraturas de fêmur em idosos no Brasil nos últimos anos. A variável GMF aponta que o gasto, em reais, com esse agravo aumentou gradativamente entre 2015 e 2019 (12,58; 14,29; 15,95; 17,56; 19,75 milhões de reais, respectivamente) e diminuiu 2020 (17,40 milhões de reais).

A análise territorial dos casos de fratura de fêmur em idosos aponta que as regiões do país apresentam divergências entre si. Em todos os anos, a região Sudeste lidera em número de casos, número de óbitos e TIF. O segundo lugar alterna entre as regiões Sul (2015, 2016 e 2020) e Nordeste (2017 e 2019). Em quarto e quinto lugar, aparecerem as regiões CentroOeste e Norte. Em relação ao TMPH, as regiões Norte e Nordeste aparecem em primeiro e segundo lugar, respectivamente, em todos os anos, ficando acima da média nacional em todos os anos estudados. Considerando os GT do SUS, em todos os anos, as regiões que mais gastaram com internações por fratura de fêmur em idosos foram as regiões Sudeste e Sul, seguido das regiões Nordeste, Centro-oeste e Norte. No entanto, o CD de cada região parece não ser proporcional ao GT - a diária mais cara, independente do ano, foi na região Sul. A tabela 1 descreve o panorama geral das fraturas de fêmur em idosos no período estudado, incluindo número de casos e óbitos, permanência hospitalar e os gastos associados. 
Figura 1. Panorama dos casos, óbitos, internação e gastos, em reais, com fratura de fêmur em idosos atendidos pelo SUS no Brasil entre 2015 e 2020 . A) Relação entre número de óbitos e taxa de mortalidade por fratura de fêmur em idosos atendidos no SUS no Brasil de 2015 a 2020 . B) Relação entre o gasto total e custo-dia, em reais, das fraturas de fêmur em idosos atendidos no SUS no Brasil de 2015 a 2020

\section{A}

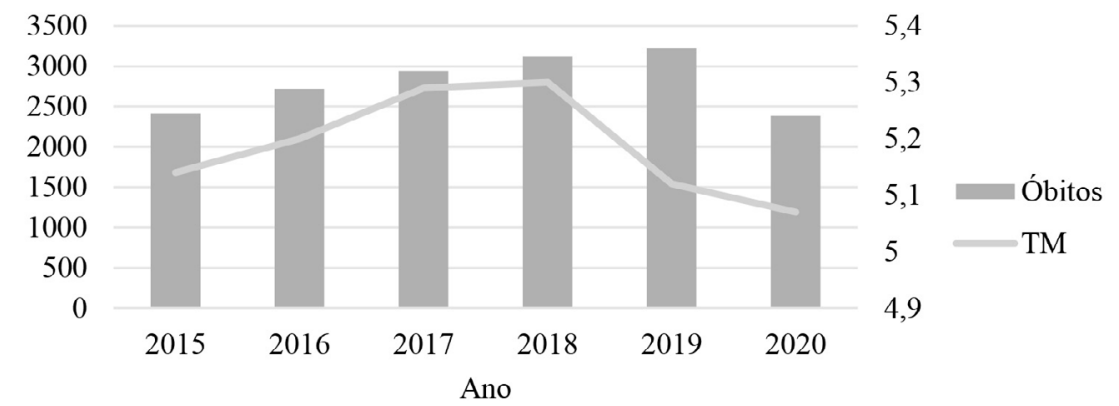

B

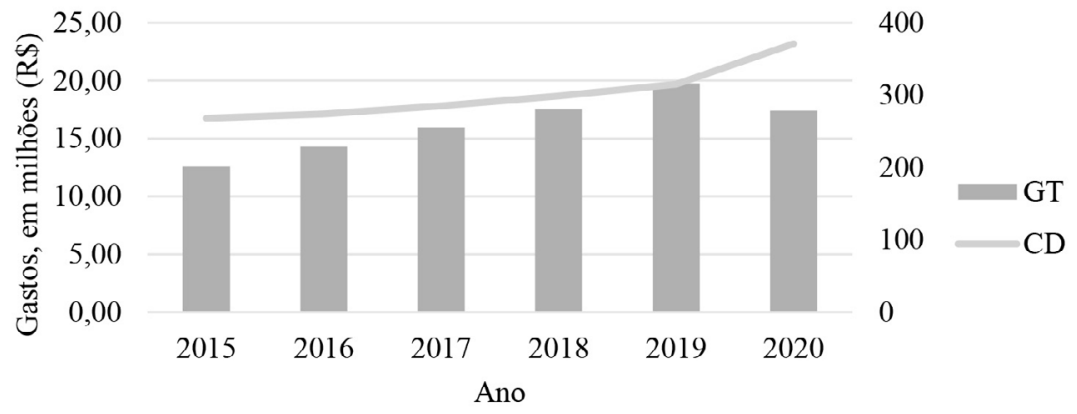

TM: taxa de mortalidade; GT: gasto total, em reais; CD: custo-dia, em reais. 
Tabela 1. Panorama dos casos, óbitos, permanência hospitalar e gastos, em reais, associados às fraturas de fêmur em idosos atendidos pelo SUS no Brasil, por região, de 2015 a 2020

\begin{tabular}{|c|c|c|c|c|c|c|c|c|c|c|c|c|}
\hline \multicolumn{13}{|c|}{ CASOS E ÓBITOS } \\
\hline Ano & \multicolumn{2}{|l|}{2015} & \multicolumn{2}{|l|}{2016} & \multicolumn{2}{|l|}{2017} & \multicolumn{2}{|l|}{2018} & \multicolumn{2}{|l|}{2019} & \multicolumn{2}{|l|}{2020} \\
\hline Região & Casos & Óbitos & Casos & Óbitos & Casos & Óbitos & Casos & Óbitos & Casos & Óbitos & Casos & Óbitos \\
\hline $\mathbf{N}$ & 1.807 & 65 & 1.904 & 69 & 1.983 & 70 & 2.272 & 78 & 2.472 & 79 & 1.972 & 62 \\
\hline $\mathrm{NE}$ & 8.751 & 304 & 9.148 & 315 & 10.446 & 382 & 10.915 & 423 & 12.215 & 439 & 9.965 & 350 \\
\hline $\mathrm{CO}$ & 2.917 & 138 & 3.363 & 158 & 3.635 & 167 & 3.984 & 215 & 4.667 & 173 & 3.537 & 140 \\
\hline SE & 24.217 & 1.363 & 27.374 & 1.592 & 28.797 & 1.736 & 30.004 & 1.825 & 32.115 & 1.951 & 26.859 & 1.402 \\
\hline $\mathbf{S}$ & 9.446 & 545 & 10.341 & 587 & 10.173 & 588 & 11.123 & 579 & 11.633 & 579 & 10.153 & 430 \\
\hline Brasil & 47.138 & 2.415 & 52.130 & 2.721 & 55.034 & 2.943 & 58.298 & 3.120 & 63.102 & 3.221 & 52.306 & 2.384 \\
\hline \multicolumn{13}{|c|}{ PERMANÊNCIA HOSPITALAR ${ }^{a}$} \\
\hline Ano & \multicolumn{2}{|c|}{2015} & \multicolumn{2}{|l|}{2016} & \multicolumn{2}{|l|}{2017} & \multicolumn{2}{|l|}{2018} & \multicolumn{2}{|l|}{2019} & \multicolumn{2}{|l|}{2020} \\
\hline Região & Dias & Média $^{b}$ & Dias & Média & Dias & Média & Dias & Média & Dias & Média & Dias & Média \\
\hline $\mathbf{N}$ & 22.900 & 12,5 & 22.698 & 12,1 & 24.484 & 12,2 & 27.193 & 11,7 & 29.740 & 12,3 & 13.697 & 8,6 \\
\hline NE & 87.733 & 10 & 88.479 & 9,6 & 101.515 & 9,7 & 108.382 & 9,8 & 114.139 & 9,3 & 71.221 & 8 \\
\hline CO & 25.618 & 8,9 & 29.953 & 8,8 & 31.472 & 8,5 & 34.578 & 8,5 & 34.258 & 7,5 & 20.669 & 6,5 \\
\hline SE & 214.953 & 8,9 & 252.229 & 9,2 & 256.151 & 8,8 & 256.281 & 8,5 & 265.887 & 8,3 & 171.066 & 7 \\
\hline 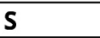 & 77.147 & 8,2 & 80.563 & 7,8 & 78.829 & 7,6 & 90.454 & 8 & 89.554 & 7.7 & 56.312 & 6.5 \\
\hline Brasil & 427.995 & 9,1 & 473.922 & 9,1 & 492.451 & 8,8 & 516.888 & 8,8 & 533.578 & 8,5 & 332.965 & 7,1 \\
\hline \multicolumn{13}{|c|}{ 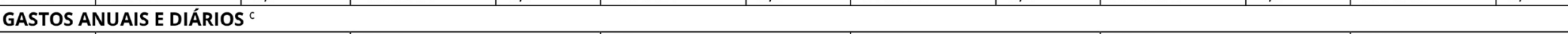 } \\
\hline Ano & \multicolumn{2}{|c|}{2015} & \multicolumn{2}{|l|}{2016} & \multicolumn{2}{|l|}{2017} & \multicolumn{2}{|l|}{2018} & \multicolumn{2}{|l|}{2019} & \multicolumn{2}{|l|}{2020} \\
\hline Região & Anual & Diário $^{d}$ & Anual & Diário & Anual & Diário & Anual & Diário & Anual & Diário & Anual & Diário \\
\hline $\mathbf{N}$ & 339.214 & 185 & 433.460 & 231 & 453.680 & 226 & 542.921 & 234 & 511.313 & 211 & 487.050 & 306 \\
\hline NE & 1.888 .641 & 216 & 2.143 .460 & 233 & 2.476 .481 & 237 & 2.856 .048 & 258 & 3.376 .216 & 275 & 2.851 .626 & 320 \\
\hline CO & 718.489 & 250 & 860.823 & 251 & 983.955 & 266 & 1.113 .354 & 274 & 1.423 .389 & 312 & 1.126 .078 & 354 \\
\hline SE & 6.690 .920 & 277 & 7.436 .442 & 271 & 8.389 .645 & 288 & 9.252 .202 & 306 & 10.393.965 & 324 & 9.268 .609 & 379 \\
\hline 5 & 3.104 .236 & 330 & 3.626 .289 & 351 & 3.771 .978 & 364 & 4.015 .553 & 355 & 4.323 .920 & 372 & 3.750 .017 & 433 \\
\hline Brasil & 12.585 .172 & 268 & 14.296 .445 & 274 & 15.956 .396 & 285 & 17.565 .124 & 299 & 19.756 .174 & 315 & 17.405 .144 & 371 \\
\hline
\end{tabular}

a) Em dias de internação. b) Valor médio por paciente. c) Valores em reais, arredondados. d) Custo-dia (CD). N = Região Norte; NE = Região Nordeste; CO = Região Centro-oeste; $S E$ = Região Sudeste; $S=$ Região Sul. 


\section{Discussão}

Este estudo objetivou investigar retrospectivamente o total de casos de fratura de fêmur em idosos realizando um comparativo entre as regiões brasileiras, a análise foi direcionada aos custos hospitalares, tempo de internação e número de óbitos. A relevância desta investigação refere-se aos impactos e consequências das fraturas de fêmur, tanto para a população idosa, quanto para o sistema de saúde. Elas são responsáveis por reduzir a expectativa de vida, gerar incapacidade física nos indivíduos acometidos e em sua grande maioria são precedidas pelo evento queda. ${ }^{12}$ Esta abordagem já foi investigada em estudos no Brasil, porém nenhum estudo contemplou todas as variáveis trazidas neste estudo ou com a visão analógica nacional entre as regiões.

Ao tentar compreender as diferenças regionais para os casos de fratura de fêmur em idosos no Brasil, observou-se uma discrepância, em que a região com mais casos foi a região sudeste, esta foi também a região que mais teve gastos em comparação às outras regiões brasileiras. No entanto, quando analisado o tempo médio de internação hospitalar, as regiões Norte e Nordeste, respectivamente, ficaram à frente da região Sudeste. Esta é a região mais populosa do país, fato que pode ter contribuído para o maior número de casos de fratura de fêmur em idosos, onde os estados do Rio de Janeiro, São Paulo e Minas Gerais concentram o maior percentual de idosos do Brasil. $\underline{13}$

Uma pesquisa identificou que na região Norte do país as internações por fratura de fêmur em idosos são mais incidentes no sexo feminino, com idade superior a 80 anos e metade dos óbitos totais por fraturas ósseas entre os anos de 2015 a 2019 em indivíduos com mais de 60 anos correspondem as fraturas de fêmur. ${ }^{14}$ Esses dados vão de encontro aos achados de um estudo que identificou o perfil epidemiológico de fraturas de fêmur em idosos no estado do Espírito Santo. $\frac{15}{}$ Ambos os estudos mostram perfis semeIhantes dos idosos vítimas de fratura de fêmur em regiões distintas do país, no entanto, nosso estudo verificou que há diferença no tempo médio de internação hospitalar pelo mesmo quadro clínico, onde a região Norte teve maior tempo médio. Este fato pode estar relacionado ao maior acesso aos serviços de saúde nas regiões Sudeste e Sul do país, bem como serviços com melhor infraestrutura, o que poderia acelerar o tempo de recuperação, contribuindo para alta hospitalar mais rápida em comparação às outras regiões brasileiras. $\frac{16}{}$

Chama atenção o fato de que os maiores gastos com fratura de fêmur em idosos ocorreram nas regiões Sudeste e Sul, enquanto essas mesmas regiões tiveram menor tempo de internação hospitalar, em comparação às regiões Norte e Nordeste. Esses dados são semelhantes ao de um estudo que verificou a ocorrência de fratura de fêmur no período de 2008 a 2018, onde a maior ocorrência de fraturas de fêmur em idosos no país se deu na região Sudeste e durante o inverno, além disso, a pesquisa demonstrou que os maiores custos com fraturas de fêmur ocorreram na região Sudeste, enquanto a região Norte teve os menores gastos ${ }^{17}$, este fato pode estar relacionado com o menor quantitativo de fraturas de fêmur em idosos na referida região.

Os custos com internações por fratura de fêmur em idosos correspondem a $2 \%$ de todo o custo com internações de idosos no Brasil. No ano de 2008 a região Norte gastou 1,2\% com internações hospitalares por fratura de fêmur, em que $0,5 \%$ dos idosos internados tinham diagnóstico de fratura de fêmur. Este número consideravelmente menor em comparação às demais regiões brasileiras, pode estar relacionado com a menor densidade populacional. $\frac{18}{}$

O prolongamento do tempo de internação hospitalar contribui negativamente para os idosos que sofreram fratura de fêmur, colaborando para o surgimento de agravos adquiridos no ambiente hospitalar, tais como, processos infecciosos do trato urinário, pneumonia, lesão por pressão e sepse, além de elevar a taxa de mortalidade. ${ }^{19} \mathrm{Um}$ estudo realizado em um hospital escola do Paraná mostrou que um prolongado tempo de internação hospitalar aguardando correção cirúrgica esteve associado ao surgimento de lesão por pressão e aumento da taxa de mortalidade, onde um tempo de espera pelo procedimento cirúrgico superior a oito dias esteve associado ao desfecho óbito. 20 Em nosso estudo, as maiores taxas de óbitos por fratura de fêmur foram registradas na região Sudeste, seguido da região Sul, enquanto que as regiões Centro-oeste e Norte corresponderam às menores taxas. A região Sudeste foi a que teve maior tempo total de internação hospitalar, fato este que pode ter contribuído para as maiores taxas de óbitos nessa região. 
A mortalidade de idosos com diagnóstico de fratura de fêmur possui íntima relação com a presença de comorbidades, tais como disfunção hepática e sarcopenia ${ }^{21}$, além de, o maior tempo de internação hospitalar, uma idade superior a 81 anos e sexo feminino contribuir para aumento na taxa de mortalidade. $?$ A fratura de fêmur isolada é um fator que contribui para a mortalidade e a densidade mineral óssea diminui cerca de 40\% após 6 meses da ocorrência da fratura, em indivíduos idosos isso contribui para aumento da fragilidade. .22

Um estudo de série temporal identificou que de 2008 a 2018 houve um aumento de $76,9 \%$ nos registros de hospitalização por fratura de fêmur em idosos, em que a taxa de mortalidade no período foi de $5 \%$, a região Sudeste deteve o maior índice (5,53\%), o maior tempo de hospitalização foi na região Centro-oeste e os maiores custos na região Sul. ${ }^{23} \mathrm{Em}$ nosso estudo, a região Sudeste deteve o maior número total de óbito para fraturas de fêmur, no entanto, o menor TMPH para o quadro. Este fato pode ter relação com a concentração de riquezas nas regiões Sudeste e Sul do país, que proporcionaram melhorias nos serviços de saúde e maior acesso da população a estes serviços. ${ }^{16}$ Os óbitos por fratura de fêmur em idosos tem significante representatividade no país e forte associação com fatores já bem descritos na literatura, como a presença de comorbidades e o prolongamento do tempo de internação hospitalar24; dessa forma, faz-se necessário otimizar tais questões, a fim de reduzir a taxa de óbitos por esse quadro. $\frac{25}{5}$

A abordagem ecológica permitiu realizar a analogia entre as regiões brasileiras, pois essa visão da coletividade pode visualizar a complexidade do sistema e da saúde pública sobre as populações, questões menos possíveis de serem contempladas em nível individual. Neste sentido, o estudo ecológico pode ser usado como marco explicativo dos problemas de saúde em questão para determinar uma intervenção de saúde a serem propostas pelas políticas públicas de saúde. Dessa forma mapear onde se faz necessário o investimento na assistência em saúde, em qual região há mais carência e qual nível de atenção à saúde deve ser priorizado seja a nível primário, secundário, terciário e quaternário. $\underline{\underline{26}}$
Otimizar a reabilitação de um indivíduo idoso em recuperação após sofrer uma fratura de fêmur pode reduzir o seu tempo de internação hospitalar. A fisioterapia deve ser iniciada tão logo a fratura seja corrigida e o tratamento deve ser continuado mesmo após a alta hospitalar. ${ }^{27}$ A mobilização precoce de idosos vítimas de fratura de fêmur é recomendada após a realização do procedimento cirúrgico para correção da fratura e está associada a redução da mortalidade 6 a 12 meses após a fratura de fêmur, além disso, os pacientes que caminham dentro de 10 dias após o procedimento cirúrgico apresentam menores índices de mortalidade. $\underline{28}$ Tudo isso impacta no tempo de internação hospitalar, na recuperação funcional do idoso, ainda que haja redução da autonomia funcional do indivíduo no pós-operatório, e na redução de demanda de cuidadores após a alta hospitalar.., 29

Este estudo ressalta a necessidade de ações de saúde que devem também ser direcionadas à médio e longo prazo, pois após a ocorrência da fratura e com o processo de envelhecimento, há um aumento da propensão a quedas acrescida com o medo de cair, fatores que repercutem na capacidade funcional e sintomas de estresse pós-traumático relacionados à queda.

Dentre as limitações do estudo, os dados não permitem realizar a associação de causa-efeito, o maior número de casos e os maiores custos na região Sudeste pode ser inferido da maior densidade populacional na região.

\section{Conclusão}

As fraturas de fêmur em idosos continuam a ocorrer de maneira frequente no país e há uma discrepância, seja no número de casos, nos custos e no total de óbitos entre as regiões brasileiras. A maior densidade populacional em determinadas regiões, como Sudeste e Sul, pode contribuir para os maiores números toais de fraturas de fêmur em idosos, haja vista que estes também são maioria nessas regiões em comparação as demais. 
Observou-se um aumento do número de casos de fratura de fêmur em idosos no país nos quatro primeiros anos estudados e uma queda em 2020, fato inverso ao observado no TMPH, que decresceu entre 2015 a 2020. Os custos com tal diagnóstico aumentaram gradativamente entre 2015 e 2019 e apresentou decréscimo em 2020. O número de óbitos se manteve crescente nos três primeiros anos, reduzindo nos dois últimos anos analisados. A região Sudeste detém os maiores números de casos, óbitos e TIF, e o maior TMPH foi observado nas regiões Norte e Nordeste, estas, junto com a região Centro-oeste, foram as que apresentaram os menores gastos com fraturas de fêmur em idosos, em contrapartida os maiores gastos foram observados nas regiões Sudeste e Sul.

As discrepâncias para o quadro de fratura de fêmur em idosos no país merecem atenção individualizada e o direcionamento de políticas públicas de saúde específicas para cada região.

\section{Contribuições dos autores}

Silva JCA, Ribeiro MDA, Silva LN e Oliveira SB participaram da concepção, análise dos dados, redação e revisão crítica do manuscrito. Pinheiro HA e Bezerra LMA participaram da revisão crítica do manuscrito.

\section{Conflitos de interesses}

Nenhum conflito financeiro, legal ou político envolvendo terceiros (governo, empresas e fundações privadas, etc.) foi declarado para nenhum aspecto do trabalho submetido (incluindo, mas não se limitando a subvenções e financiamentos, participação em conselho consultivo, desenho de estudo, preparação de manuscrito, análise estatística, etc.).

\section{Referências}

1. Diniz JE. Envelhecimento populacional continua e não há perigo de um geronticídio [Internet]. Laboratório de demografia e estudos populacionais. Disponível em: https://www.ufjf.br/ ladem/2020/06/21/envelhecimento-populacional-continua-enao-ha-perigo-de-um-geronticidio-artigo-de-jose-eustaquio-dinizalves/

2. Soares DS, Mello LM, Silva AS, Martinez EZ, Nunes AA. Fraturas de fêmur em idosos no Brasil: análise espaço-temporal de 2008 a 2012. Cad. Saúde Pública. 2014;30(12):2669-78. http://dx.doi. org/10.1590/0102-311X00218113
3. Franco LG, Kindermann AL, Tramujas L, Kock KS. Fatores associados à mortalidade em idosos hospitalizados por fratura de fêmur. Rev Bras Ort. 2016;51(5):509-14. http://dx.doi. org/10.1016/j.rbo.2015.10.009

4. Torres MRS, Oliveira LB, Peixoto MI. Associação entre sarcopenia e história de fraturas em pacientes idosos com diabetes tipo 2. Medicina. 2020;53(4):389-97. https://doi. org/10.11606/issn.2176-7262.v53i4p389-397

5. Macedo GG, Teixeira TRG, Ganem G, Daltro GC, Faleiro TB, Rosário DAV, et al. Fraturas de fêmur em idosos: um problema de saúde pública no Brasil. REAC/EJSC. 2019;6(e112):1-7. https://doi. org/10.25248/reac.e1112.2019

6. Guerra MTE, Viana RD, Feil L, Feron ET, Maboni J, Vargas ASG. Mortalidade em um ano de pacientes idosos com fratura do quadril tratados cirurgicamente num hospital do sul do Brasil. Rev Bras Ort. 2017;52(1):17-23. http://dx.doi.org/10.1016/j. rbo.2016.04.005

7. Moreira RS, Souza JG, Siqueira AR, Xavier MD, Oliveira $\mathrm{SP}$, Bauman CD. Mortalidade em idosos com fratura de fêmur proximal em um hospital universitário. REAS/EJCH. 2021;13(1):e6382. https://doi.org/10.25248/reas.e6382.2021

8. Porto AO, Leal CBM, Rios MA, Fernandes TSS, Fernandes ESF, Ferreira RBS. Características sociodemográficas e custo de hospitalização por fratura de fêmur em idosos na Bahia. Journal Health NPEPS. 2019;4(2):297-309. http://dx.doi. org/10.30681/2526101038233823

9. Santos LES, Santos VV, Naziazeno SDS, Santos LS. Fatores causais associados à fratura de fêmur em idosos. Ciências Biológicas e de Saúde Unit [Internet]. 2021;6(3):121-34. Disponível em: https://periodicos.set.edu.br/cadernobiologicas/article/ view/9865/4460

10. Lima-costa MF, Barreto SM. Tipos de estudos epidemiológicos: conceitos básicos e aplicações na área do envelhecimento. Epidemiol. Serv. Saúde. 2003;12(4):189-201. http://dx.doi. org/10.5123/S1679-49742003000400003

11. Ministério da Saúde. DATASUS [Internet]. Disponível em: http://tabnet.datasus.gov.br/cgi/deftohtm.exe?sih/cnv/miuf.def

12. Neto AADS, Silva PR, Nascimento $C H O$, Souza CS. Fratura de fêmur em idosos hospitalizados: revisão integrativa. Ciências Biológicas e de Saúde Unit [Internet]. 2017;4(2):203-14. Disponível em: https://periodicos.set.edu.br/fitsbiosaude/article/ view/4526/2615

13. Pala LOO, Ferreira EB, Petrini J. O crescimento do percentual de idosos na região sudeste: uma aplicação via modelo linear misto. Sigmae [Internet]. 2019;8(2):180-90. Disponível em: http:// publicacoes.unifal-mg.edu.br/revistas/index.php/sigmae/article/ view/953/630 
14. Leite CO, Tavares JP, Botelho KKP, Cesar FCR, Rodrigues ESR, Cláudio ES. Internações por fratura de fêmur em idosos na região Norte do Brasil. Revista Amazônia Science \& Health [Internet]. 2020;8(4):10-8. Disponível em: http://ojs.unirg.edu.br/index. php/2/article/view/3202/1699

15. Mielke J, Vicente CR. Perfil epidemiológico e mortes por fratura de fêmur em idosos residentes no estado do Espírito Santo de 2010 a 2017. Rev. Bras. Pesq. Saúde. 2020;22(4):32-7. https://doi. org/10.47456/rbps.v22i4.21767

16. Albuquerque MV, Viana ALA, Lima LD, Ferreira MP, Fusaro ER, Iozzi FL. Desigualdades regionais na saúde: mudanças observadas no Brasil de 2000 a 2016. Ciênc Saúde Colet. 2017;22(4):1055-64. https://doi.org/10.1590/1413-81232017224.26862016

17. Silva DA, Pereira JF, Gonçalo MV, Nascimento NM, Oliveira CMS. Levantamento de fratura de fêmur e óbito em pessoas idosas: uma análise quantitativa nas regiões brasileiras. Revista Kairós Gerontologia. 2021;23(4):415-29. http://dx.doi. org/10.23925/2176-901X.2020v23i4p415-429

18. Bortolon PC, Andrade CLT, Andrade CAF. O perfil das internações do SUS para fratura osteoporótica de fêmur em idosos no Brasil: uma descrição do triênio 2006-2008. Cad. Saúde Pública. 2011;27(4):733-42. https://doi.org/10.1590/S0102$311 \times 2011000400012$

19. Lopes FPRA, Silva EC, Melo LB, Ferreira HS. O papel do enfermeiro ao paciente idoso com fraturas de fêmur. Revista Multidebates [Internet]. 2021;5(2):153-64. Disponível em: http://revista.faculdadeitop.edu.br/index.php/revista/article/ view/338/329

20. Alcantara C, Dellaroza MSG, Ribeiro RP, Carvalho CJA. Fratura de fêmur nos idosos: tempo de espera cirúrgica e desfecho da hospitalização. Cienc Cuid Saude [Internet]. 2021;20:e54726. Disponível em: https://periodicos.uem.br/ojs/index.php/ CiencCuidSaude/article/view/54726

21. Monnerat VBM, Silva CPO, Ramos AMP, Mathias MB, Novellino $P$, Fiorelli SKA, et al. Avaliação da mortalidade no pós-operatório de fraturas de fêmur em idosos com comorbidades prévias. Fisioterapia Brasil [Internet]. 2021;22(1):49-60. Disponível em: https://portalatlanticaeditora.com.br/index.php/fisioterapiabrasil/ article/view/4514
22. Merloz P. Optimization of perioperative management of proximal femoral fracture in the elderly. Orthop Traumatol Surg Res. 2018;104:S25-230. https://doi.org/10.1016/j.otsr.2017.04.020

23. Peterle VCU, Geber Junior JC, Darwin Junior W, Lima AV, Bezerra Junior PE, Novaes MRCG. Indicadores de morbidade e mortalidade por fraturas de fêmur em idosos: análise de uma década em hospitais brasileiros. Acta Ortop Bras. 2020;28(3):1428. http://dx.doi.org/10.1590/1413-785220202803228393

24. Antunes Filho J, Silva ALC, Mendes Junior AF, Pereira FJC, Oppe IG, Loures EA. Fatores preditivos de morte após cirurgia para tratamento de fratura proximal de F,mêmur. Rev Bras Ortop. 2019;54(4):402-7. https://doi.org/10.1055/s-0039-1692179

25. Oliveira CC, Borba VZC. Epidemiologia das fraturas de fêmur em idosos e seu custo para o estado do Paraná, Brasil. Acta Ortop Bras. 2017;25(4):155-8. http://dx.doi.org/10.1590/1413$\underline{785220172504168827}$

26. Brevidelli MM, Freitas FCG. Estudo ecológico sobre o desenvolvimento da saúde no Brasil. Ciênc. saúde coletiva. 2012;17(9):2471-80. https://www.scielosp.org/article/csc/2012. v17n9/2471-2480/

27. Borges AEA, Liberali R. Perfil epidemiológico de idosos com fraturas diversas, atendidos nos hospitais brasileiros: uma revisão de literatura. Rev Kairós. 2018;21(4):353-69. http://dx.doi. org/10.23925/2176-901X.2018v21i4p353-369

28. Aprato A, Bechis M, Buzzone M, Bistolfi A, Daghino W, Massè A. No rest for elderly fêmur fracture patients: early surgery and early ambulation decrease mortality. J Orthop Traumatol. 2020;21:12. https://doi.org/10.1186/s10195-020-00550-y

29. Petros RSB, Ferreira PEV, Petros RSB. Influência das fraturas do fêmur proximal na autonomia e mortalidade dos pacientes idosos submetidos a osteossíntese com haste cefalomedular. Rev Bras Ortop. 2017;52(suppl 1):57-62. http://dx.doi.org/10.1016/j. rbo.2017.06.011

30. Dyer SM, Crotty M, Fairhall N, Magaziner J, Beaupre LA, Cameron ID, et al. A critical review of the long-term disability outcomes following hip fracture. BMC Geriatrics. 2016;16:158. https://doi.org/10.1186/s12877-016-0332-0

31. Eckert T, Kampe K, Kohler M, Albrecht D, Büchele G, Hauer $\mathrm{K}$, et al. Correlates of fear of falling and falls efficacy in geriatric patients recovering from hip/pelvic fracture. Clin Rehabil. 2020;34(3):416-25. https://doi.org/10.1177/0269215519891233 